\title{
Disseminated cutaneous Mycobacterium tuberculosis infection in a patient with AIDS
}

\author{
E L Corbett, I Crossley, K M De Cock, R F Miller
}

Individuals infected with the human immunodeficiency virus (HIV) are at an increased risk of both pulmonary and extrapulmonary tuberculosis. ${ }^{1}$ Disseminated cutaneous tuberculosis is rare, but has been reported in four HIV-positive patients, all of whom also had pulmonary infection. ${ }^{2-5}$ In this report we describe an HIV-infected patient with a febrile illness and an abnormal chest radiograph who developed widespread cutaneous tuberculous pustules following a lymph node biopsy on the previous day.

(Genitourin Med 1995;71:308-310)

Keywords: tuberculosis; AIDs; cutaneous

HIVIAIDS Unit,

Camden and Islington

Community Health

Services NHS Trust,

The Middlesex

Hospital, Mortimer

Street, London

W1N 8AA, UK

E L Corbett

I Crossley

K M De Cock

R F Miller

Accepted for publication 15 June 1995

Figure 1 CT scan of the thorax showing hilar and mediastinal

lymphadenopathy. A mas is seen arising at the right hilum extending into the middle lobe. AZT for the previous two years, otherwise he had been well. He recalled BCG vaccination $\stackrel{5}{\circ}$ as a school child, but had no apparent scar. On admission he was noted to have a small palpable right supraclavicular lymphnode, $a_{\overparen{\Phi}}$ palpable liver edge and splenic tip and was pyrexial, with temperature $39 \cdot 7^{\circ} \mathrm{C}$. Investi- gations showed that he was anaemic (haemo- $\overrightarrow{0}$ globin $=9.4 \mathrm{~g} \mathrm{dl}^{-1}$ ), and had a low white cell count, $3.4 \times 10^{9} \mathrm{ml}^{-1}$, CD4+ lymphocyteo count $=0.30 \times 10^{9} \mathrm{ml}^{-1}$ (normal range $=\stackrel{\rho}{=}$ $0.35-2.2 \times 10^{9} \mathrm{ml}^{-1}$ ). Liver function tests were normal apart from an albumin of $31 \mathrm{gl}^{-1} \dot{\mathrm{c}}$ (normal range $=35-33 \mathrm{gl}^{-1}$ ). A chest radio- $\omega$ graph showed right hilar enlargement and left ${ }_{\circ}^{\infty}$ upper zone interstitial shadowing compatible with his previous $P$ carinii pneumonia. At $\vec{O}$ fibreoptic bronchoscopy, no anatomical abnormality was noted. Auramine and methenamine staining of bronchoalveolar lavage fluid from the left upper lobe were negative. $\&$ Samples of blood, stool, urine and broncho-. alveolar fluid were cultured for mycobacteria.

Over the course of the next two weeks the patient's condition deteriorated markedlyo with loss of $7 \mathrm{~kg}$ in weight, increase in size of the supraclavicular lymph node to $3 \times 2 \mathrm{~cm}$ and clinical and radiographic evidence of $\mathrm{a}$ 을 right middle lobe collapse/consolidation. $\frac{\Im}{5}$ Spiral computed tomography (CT) of the

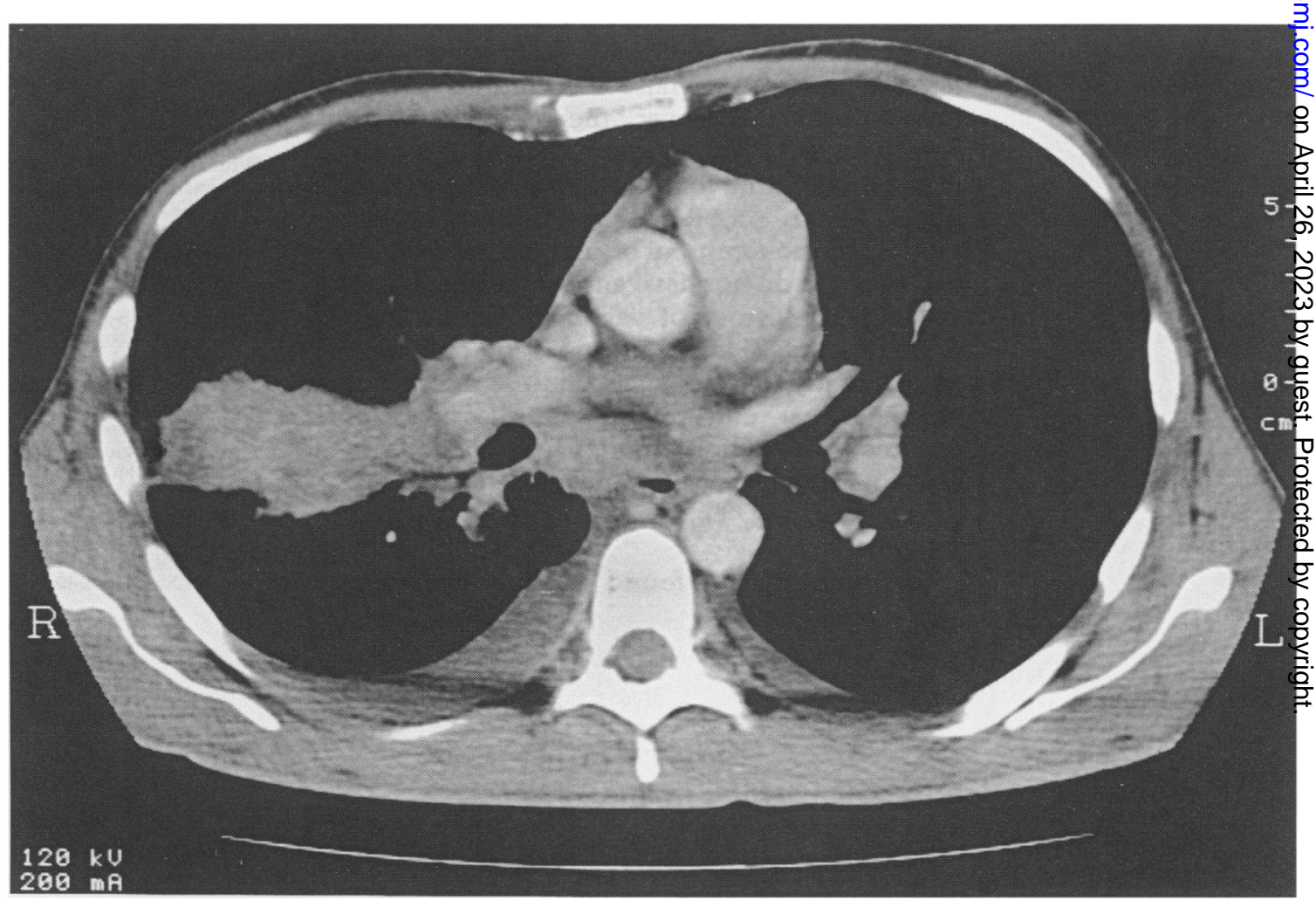


chest showed marked and inhomogeneous hilar and mediastinal lymphadenopathy with peripheral contrast enhancement, compression of the right main bronchus and a mass extending from the right hilum into the right middle lobe (fig 1). An abdominal ultrasound scan showed a $16 \mathrm{~cm}$ spleen and no retroperitoneal lymphadenopathy. The time course of the illness and the CT scan appearances were felt to be most consistent with an aggressive lymphoma. A bone marrow aspirate and trephine were nondiagnostic. On the 16th day of admission the enlarged supraclavicular lymph node was biopsied. On the morning following surgery the patient developed a rash which consisted of sparse $2-3 \mathrm{~mm}$ pustules on an erythematous base distributed over the trunk and thighs (fig 2). Microscopy of an auramine stained sample of pus from the skin lesions showed mycobacteria and histological examination of the lymph node biopsy also showed mycobacteria, which were present in large numbers. Culture from lavage fluid, sputum, stool, urine, bone marrow, skin and lymphnode grew Mycobacterium tuberculosis which was subsequently found to be isoniazid resistant.

Treatment was begun with isoniazid, rifampicin, ethambutol and pyrazinamide in conventional doses and the patient was isolated and notified to the Director of Public

Figure 2 Pustular rash over the trunk.
Health. Isoniazid was discontinued when resistance was demonstrated. He made an uneventful recovery with resolution of the pustules into dry scabs over 7-10 days, and a more gradual improvement of the chest radiographic abnormalities. He was discharged home two weeks after the start of antituberculous treatment and remains well with sustained weight gain four months later.

\section{Discussion}

The clinical and radiographic presentation of tuberculosis is frequently atypical in patients with HIV. ${ }^{167}$ Clinically apparent extrapulmonary disease is more common, and chest radiograph appearances are often non specific. ${ }^{16}$ Tuberculosis may present as a rapidly progressive disease in immunosuppressed patients. ${ }^{1}$ Haematogenous spread of tuberculosis to the skin resulting in a generalised eruption, which is known as tuberculosis cutis miliaris acuta generalisata, is a rare condition that has been described in the context of both primary infection and following reactivation of an endogenous focus. ${ }^{8}$ Prior to the HIV epidemic, acute milary tuberculosis of the skin was most commonly seen in children, occurred most often following a viral exantham or severe bacterial infection, and had a high short term mortality rate. ${ }^{89}$ The rash of tuberculosis cutis miliaris acuta generalisata is quite different from other more common forms of cutaneous tuberculosis, such as scrofuloderma or lupus vulgaris, and consists of multiple pustules with a centripetal distribution. ${ }^{8910}$

Recently four published cases of cutaneous milary tuberculosis have been described in patients with AIDS. ${ }^{2-5}$ The presentation of these patients have several features in common with that of our own case. All these patients were profoundly immunosuppressed with $\mathrm{CD} 4+$ lymphocyte counts ranging between 0.16 to $0.2 \times 10^{9} \mathrm{ml}^{-1}$ and all four patients were anaemic. In three of these cases patients were admitted with a febrile illness, without a clinically obvious source, and normal initial chest radiographs apart from mild cardiomegaly in one patient and questionable mediastinal lymphadenopathy in another. The initial working diagnoses in these patients were bacterial endocarditis in two cases and $P$ carinii pneumonia in the third. The fourth patient had miliary shadowing on the admission chest radiograph and was sputum smear positive for mycobacteria, although microscopy of bronchoalveolar lavage fluid obtained two weeks earlier had been negative for acid-fast bacilli. In each of the four cases the cutaneous lesions developed suddenly while the patients were in hospital and the appearances and distribution were similar to those in our patient. All four patients were critically ill and two died; in one of the two fatal cases tuberculosis was diagnosed only at post mortem examination and in the other the diagnosis was made from a skin biopsy obtained two days before the patient's death. Diagnosis in the two surviving patients was 
made by microscopy of sputum and lavage fluid respectively.

The temporal relationship between the lymph node biopsy and the appearance of the rash in our patient suggests that haematogenous spread may have been exacerbated by handling of the node at the time of surgery, although subsequent positive cultures of $M$ tuberculosis from urine collected prior to surgery indicate that dissemination had already occurred.

The thoracic CT appearances in our patient were compatible with tuberculosis in advanced HIV disease, with massive mediastinal and hilar lymphadenopathy together with a parenchymal infiltrate. ${ }^{7}$ Surprisingly, despite the marked radiographic abnormalities, microscopy of the lavage fluid was negative for acid-fast bacilli. HIV positive patients with culture positive pulmonary tuberculosis are less likely to be smear positive on microscopy for acid-fast bacilli than are non-HIV infected patients, even in the presence of extensive chest radiograph abnormalities. ${ }^{11}$

In conclusion, although our case is not unique, it illustrates the need to maintain a high index of suspicion for disseminated tuberculosis in HIV-infected patients, and also underlines the high diagnostic yield from microscopy and culture of any unusual rashes in this patient group.

1 Foley NM and Miller RF. Tuberculosis and HIV infection: is "the white plague" up and coming? $\mathcal{F}$ Infect 1993; 26:39-43.

2 Stack RJ, Bickley LK, Coppel IG. Miliary tuberculosis presenting as skin lesions in a patient with acquired immunodeficiency syndrome. 7 Am Acad Dermatol 1990; 23:1031-5.

3 Rohatgi PK, Palazzolo JV, Saini NB. Acute miliary tuberculosis of the skin in acquired immunodeficiency synculosis of the skin in acquired immunodeficie

4 Bassiri A, Chan NB, McLeod A, et al. Disseminated cutaneous infection due to Mycobacterium tuberculosis in person with AIDS. Can Med Assoc $\mathcal{F} 1993 ; 148: 577-8$.

5 Inwald D, Nelson M, Cramp M, et al. Cutaneous manifestations of mycobacterial infection in patients with AIDS Br F Dermatol 1994;130:111-14.

6 Greenberg SD, Frager D, Suster B, Walker S, et al. Active pulmonary tuberculosis in patients with AIDS; spectrum of radiographic findings (including a normal appearance). Radiology 1994;193:115-9.

7 Pastores SM, Naidich DP, Aranda CP, et al. Intrathoracic adenopathy associated with pulmonary tuberculosis in adenopathy associated with pulmonary tuberculosis in patients with human im

8 Schermer DR, Simpson CG, Haserick JR, et al. Tuberculosis cutis miliaris acuta generalisata. Report of a case in an adult and a review of the literature. Arch Dermatol 1969;99:64-9.

9 Beyt BE, Ortbals DW, Cruz DJS, et al. Cutaneous mycobacteriosis: analysis of 34 cases with a new classification of the disease. Medicine 1980;60:95-109.

10 Seghal VN, Bhattacharya SN, Jain S, et al. Cutaneous tuberculosis: the evolving scenario. Int $f$ Dermatol 1994; 33:97-104.

11 Klein NC, Duncanson FP, Lenox TH, et al. Use of mycobacterial smears in the diagnosis of pulmonary tuberculosis in AIDS/ARC patients. Chest 1989;95: 1190-2. 